work this system is used in juxtaposition with the older Schoenflies symbolism. Comparison tables of the different systems of nomenclature formerly in use are then given, and chapters on the crystal classes and translation groups and transformations follow. We then come to the main chapter dealing with the 230 space-groups, which occupies twothirds of the volume. The description accorded to each space-group is much more complete than anything else which has appeared. The coordinates of the special and general point positions are given with the corresponding point symmetries and the lattice complex for each point position. The enumeration of the symmetry elements is completed by giving the screw axes and glide planes. A list of the sub-groups follows. All this information is then summarised by means of two diagrams for each space-group, one giving the general point positions and the other the distribution of symmetry elements. These pictures are greatly clarified as a result of this dual representation. The geometrical structure factor for the general plane $(h k l)$ is next given, and it may be noted that this is the first time a complete set of structure factors for all the space groups has been published. They represent a valuable addition to the tools of research. The description of each spacegroup is completed by listing the characteristic missing spectra, or 'halving'. These lists of missing spectra are classified in the next chapter in the form of a new table by which the possible spacegroups of any crystal can be determined in a systematic manner. Two further chapters on point symmetries and lattice complexes complete the first volume.

The second volume contains tables of mathematical and physical data which are of constant use in X-ray analysis : quadratic forms, trigonometrical and exponential functions, intensity formulæ, atomic scattering factors, absorption coefficients, wave-lengths, lattice constants, atomic radii, etc., and a long chapter on graphical methods of evaluating X-ray diagrams. The tables are admirably compact, and quite indispensable to anyone carrying out serious work on crystal structure.

In an international work of this magnitude which has been so skilfully planned and executed, there is little room for criticism, and we must congratulate the editors, Sir William Bragg, Prof. M. von Laue and Prof. C. Hermann, and the international group of authors on the successful completion of their immense task. The student will certainly find some difficulty with this work because portions are by no means easy to under. stand, although with continued use their contents may become more familiar. There is perhaps a tendency to over-elaboration in certain sections of the work. Most X-ray photographs can be analysed and the results interpreted from first principles; the use of space-group theory, for example, is important chiefly because it effects an economy of thought in presenting immediately all the mathematically possible arrangements of symmetry elements compatible with the X-ray data. But if the presentation of the theory is too elaborate the resulting economy of thought may be seriously diminished, and some workers might for a time prefer to go back to first principles rather than grapple with methods that are more complex on account of their greater generality. This criticism may apply to certain sections of the first volume, or alternatively it may be said that the reviewer does not fully understand them. The two statements are to some extent equivalent.

In minor points there are inevitably a few misprints, particularly in the second volume. We understand, however, that the publishers are preparing a leaflet of corrections. It should be noted that, contrary to the statement on p. 585, Table II, p. 588, is not based on the wave-lengths of the preceding Table I. J. M. RoBertson.

\section{The Rise of Modern Physics}

By Prof. Henry Crew. Second edition. Pp. xix + $434+16$ plates. (London: Baillière, Tindall and Cox, 1935.) $18 s$.

THE original edition of this book, published in 1927, has been extended by about a hundred pages to deal with matters "that have only lately been reduced to order". Reference is given to the latest position of definitions of electrical units, research on the inertia of electricity, modern spectroscopy and the etherdrift problem. General corrections are made and up-to-date references to important original contributions to physics are embodied.

The author explains that the term 'modern' in the title of the book is used as opposed to 'ancient', and applies to the period since the time of Galileo. The essential object of the little volume is to present an informal connected introduction to physies, in the form of a history stated to represent the "irreducible minimum for one wishing to acquire a just perspective", with liberal references to and extracts from original sources. Actually, we are led up to modern physics, since sub-atomic research is introduced only very briefly; the older quantum theory goes as far as the Bohr atom, and the new quantum mechanics is held to be outside the scope of the book.

The whole forms a very readable and interesting discussion instead of the catalogue of facts of a conventional history. There are sixteen good portrait plates, sixteen simple line diagrams, and a few mathematical formulæ; when this is borne in mind, the price is, unfortunately, almost prohibitive to the general reader.
N. M. B. 